UCRL-JC-131602

PREPRINT

\title{
A High Intensity Electron Beam Ion Trap for Charge State Boosting of Radioactive Ion Beams
}

\author{
R. E. Marrs \\ D. R. Slaughter \\ This paper was prepared for submittal to the \\ 15th International Conference on the Applications of Accelerators in Research and Industry \\ Denton, TX \\ November 4-7, 1998
}

September 30, 1998

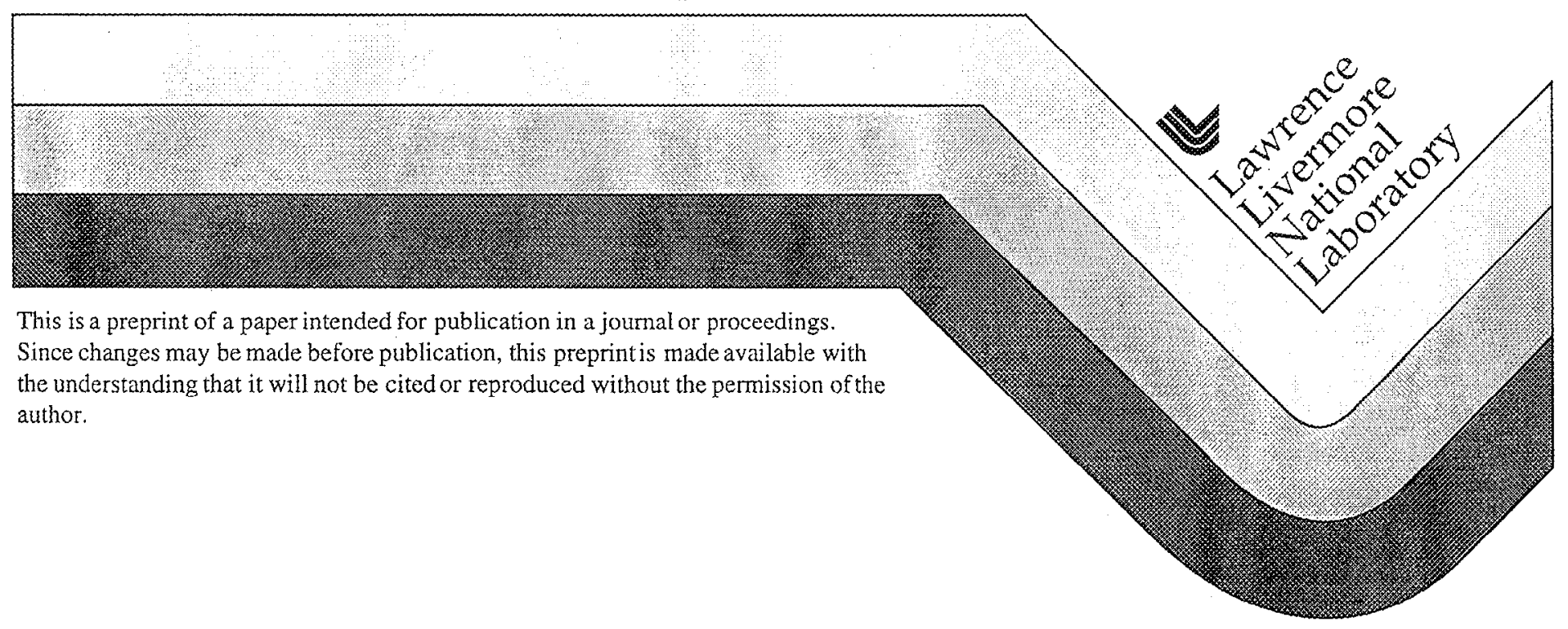




\section{DISCLAIMER}

This document was prepared as an account of work sponsored by an agency of the United States Government. Neither the United States Government nor the University of California nor any of their employees, makes any warranty, express or implied, or assumes any legal liability or responsibility for the accuracy, completeness, or usefulness of any information, apparatus, product, or process

disclosed, or represents that its use would not infringe privately owned rights. Reference herein to any specific commercial product, process, or service by trade name, trademark, manufacturer, or otherwise, does not necessarily constitute or imply its endorsement, recommendation, or favoring by the United States Government or the University of California. The views and opinions of authors expressed herein do not necessarily state or reflect those of the United States Government or the University of California, and shall not be used for advertising or product endorsement purposes. 


\title{
A High Intensity Electron Beam Ion Trap for Charge State Boosting of Radioactive Ion Beams
}

\author{
R. E. Marrs and D. R. Slaughter \\ Lawrence Livermore National Laboratory, Livermore, CA 94551.
}

\begin{abstract}
A high intensity electron beam ion trap under development at LLNL could be adapted for charge state boosting of radioactive ion beams, enabling a substantial reduction in the size and cost of a postaccelerator. We report estimates of the acceptance, ionization time, charge state distribution, emittance, and beam intensity for charge state boosting of radioactive ions in this device. The estimates imply that, for tin isotopes, over $10^{30}$ ions/s can be ionized $10 q=40+$ with an absolute emittance of approximately $1 \pi$ $\mathrm{mm}$ mrad at an energy of $30 \times q \mathrm{keV}$.
\end{abstract}

\section{INTRODUCTION}

The U. S. scientific community is considering options for the construction of a major new facility for the production and acceleration of radioactive ion beams (RIBs). The facility will be based on the ISOL concept in which radioisotopes diffuse out of a high temperature production target, are singly ionized, and pass through a mass separator. The proposed scientific program requires accelerating the radioactive ions to energies up to roughly $10 \mathrm{MeV} / \mathrm{u}$. To reach these energies in a post-accelerator of reasonable size and cost the radioactive ions must be ionized to high charge states either before acceleration or by stripping between stages of acceleration.

One option for reaching high charge states is ionization of (low energy) $q=1+$ ions in an electron beam ion source (EBIS) or trap (EBIT). In these devices ions are trapped within the space charge of an electron bearn and ionized to high charge states by electron impact. For example, isotopes of tin $(Z=50)$ could be ionized to $q=$ $40+$ or $48+$ before acceleration. A high intensity EBIT under development at LLNL may be able to satisfy the expected performance requirements of a future RIB facility with a substantial reduction in the size of the postaccelerator and an improvement in performance compared to ion stripping between acceleration stages.

\section{ELECTRON BEAM ION TRAP}

The electron beam ion trap was developed at LLNL to study $x$-ray emission from very-highly-charged ions. The ions are trapped in the space charge potential of an electron beam and confined axially by voltages applied to drift tubes or trap electrodes $(1,2)$. High charge states are reached by successive ionizing collisions with beam electrons, and the final charge state can be controlled by selecting the electron beam energy and confinement time. The EBIT concept is shown in Fig. 1. The related EBIS was developed as a source of highly-charged-ion bearns (3). The EBIT uses a higher electron beam current density and a shorter trap length than the traditional EBIS. However, the EBIT can also be operated as an ion source.

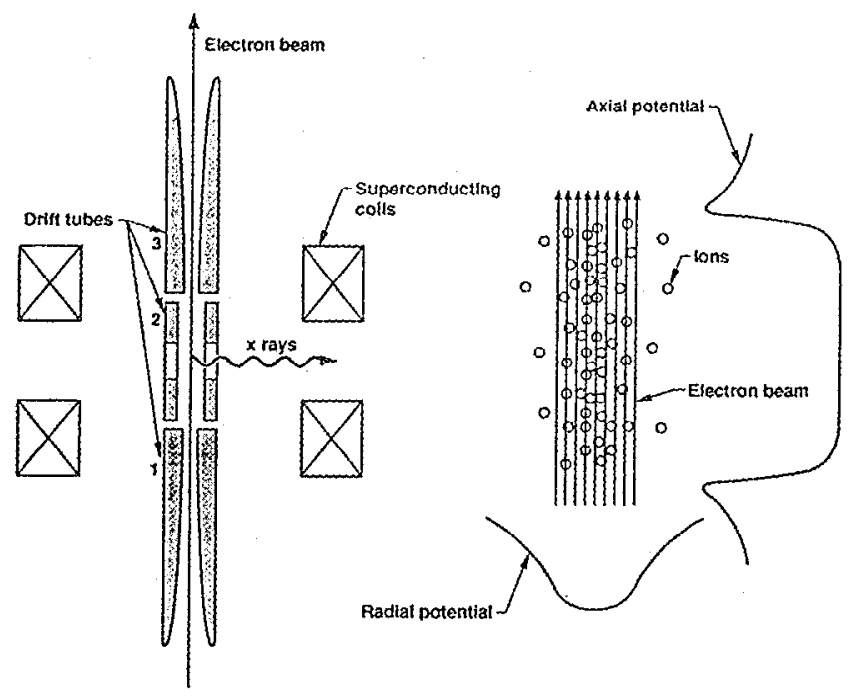

FIGURE 1. Illustration of ion confinement in an EBIT. The magnetic field compresses the electron beam, but has little effect on the ions.

A high intensity EBIT under development at LLNL is expected to increase the $\mathrm{X}$-ray emission rate from trapped ions by a factor of 100 (per $\mathrm{cm}$ of beam length), and the 
highly-charged-ion beam intensity by a factor of 1000 over that of existing EBITs. This is accomplished by roughly 10 -fold increases in the total electron beam current, the current density, and the length of the trap. Actual parameters for the LLNL high intensity EBIT are given in Table I, and a scale drawing is shown in Fig. 2.

\section{EXPECTED PERFORMANCE}

The performance of the LLNL high intensity EBIT operating as a charge state booster can be inferred from calculations and experience with existing EBIT devices. The profile of the magnetically compressed electron beam in such devices is known to be roughly Gaussian with a radius determined by the electron temperature and the magnetic field (if ary) at the cathode (4). Normally the magnetic field at the cathode is carefully zeroed with a bucking coil in order to obtain maximum electron beam compression. Electron beam profile measurements in magnetic fields up to $3 \mathrm{~T}$ confirm the predictions of theoretical models. For the high intensity EBIT operating at $6 \mathrm{~T}$ with a $5-\mathrm{A}$ electron beam from a $6.4-\mathrm{mm}$ radius cathode, theory predicts that $80 \%$ of the electron beam is within a radius of $48 \mu \mathrm{m}$. The electron beam radius is almost independent of the electron energy; however, we use an electron energy of $30 \mathrm{keV}$ for the estimates presented here.

TABLE 1. Typical parameters for the LLNL high intensity EBIT operating as a RIB charge state booster.

$\begin{array}{ll}\text { Magnetic field } & 6 \text { tesla } \\ \text { Elcctron bcam cncrgy } & 30 \mathrm{keV} \\ \text { Electron beam current } & 5 \mathrm{~A} \\ \text { Beam radius (80\% current) } & 48 \mu \mathrm{m} \\ \text { Central current density } & 1.1 \times 10^{5} \mathrm{~A} / \mathrm{cm}^{2} \\ \text { Trap length } & 25 \mathrm{~cm} \\ \text { Total electron charges } & 7.9 \times 10^{10}\end{array}$

\section{Ion Acceptance}

The acceptance of the EBIT trap is a very important parameter. It must be large enough to accommodate the emittance of the singly charged radioactive ion beam from the production source with minimal losses. Acceptance and emittance have the same units, and both are a measure of the transverse phase space volume of the ion beam. As such, they can be related to the temperature and radius of the ions in an ion source or trap. We calculate the absolute acceptance and emittance for ions entering or leaving the EBIT trap from the formula

$$
\varepsilon=\pi R_{80} \sqrt{\frac{T_{i}}{q e U}}
$$

where $R_{80}$ is the radius in the trap containing $80 \%$ of the ions, $T_{i}$ is the temperature of the trapped ions, $q$ is the ion charge state, $e$ is the elementary charge, and $U$ is the acceleration potential of the ion beam at which the emittance is specified (5). For a Maxwellian distribution of ion velocities at temperature $T_{i}$, and a Gaussian shaped radial ion distribution, the phase space defined by $\mathrm{Eq}$. (1) contains $67 \%$ of the ions.

Captured ions will have good overlap with the electron beam if the ion temperature is no larger than the space charge potential difference from the axis to the edge of the beam, which is $U_{e}=450 \mathrm{~V}$ for the parameters given in Table I, neglecting ion space charge. (The characteristic ion and electron radii for Gaussian distributions are in fact equal when $T_{i}=q e U_{\varepsilon}$.) The corresponding absolute acceptance for $T_{i}=450 \mathrm{eV}$ is $\varepsilon=5.9 \pi \mathrm{mm} \mathrm{mrad}$ at $U=30$ $\mathrm{kV}$. This is comparable to the emittance of the types of ion sources used at ISOL facilities (6).

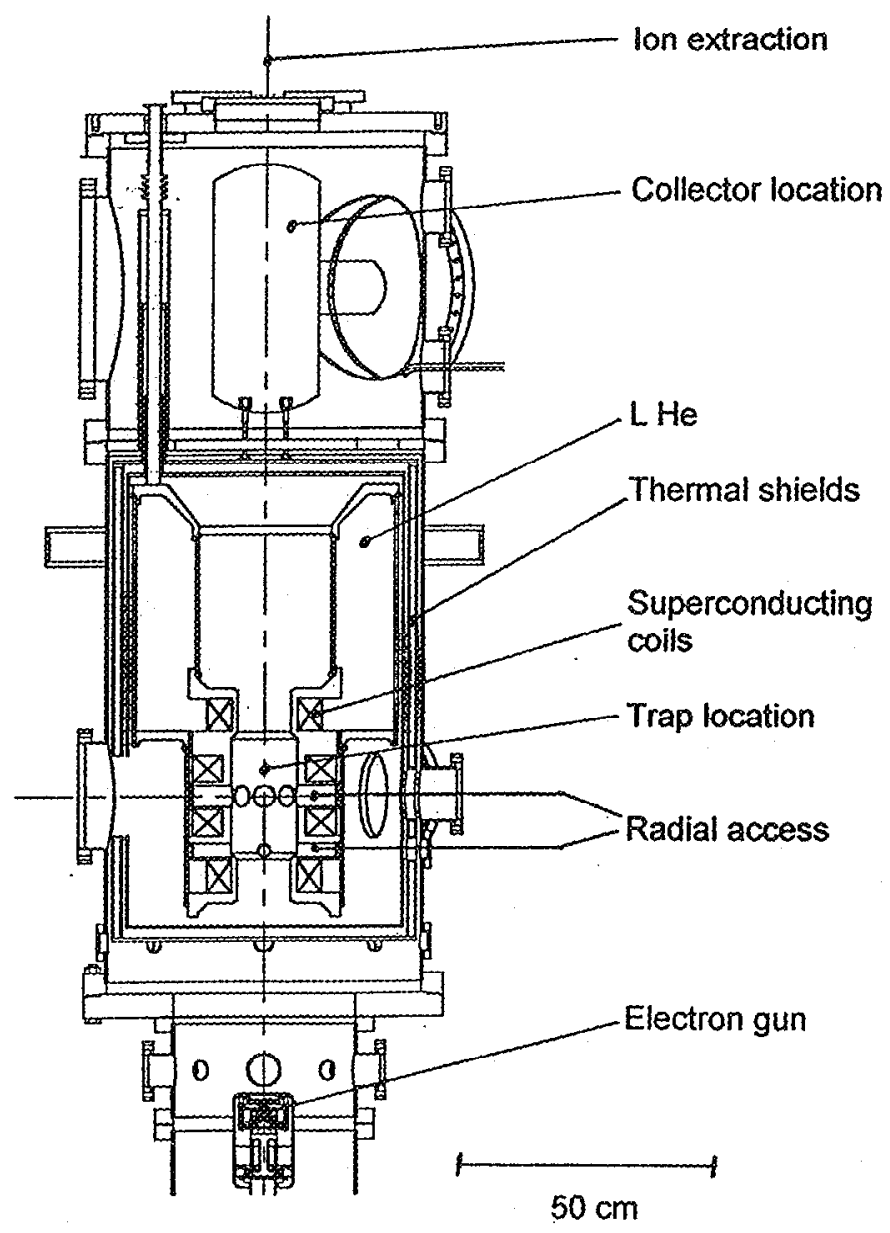

FIGURE 2. Layout of the high intensity EBIT at LLNL. 
Note that the acceptance for capture into the trap without requiring good overlap with the electron beam is much larger than the value given above because the 5-mm radius of the trap electrodes is 100 times larger than the electron beam radius, and the $4.5-\mathrm{kV}$ space charge potential at the trap electrode radius is 10 times larger than that at the beam edge. In summary, the capture efficiency for radioactive ions injected into the LLNL high intensity EBIT from ISOL type sources is expected to be close to $100 \%$ because of the deep potential well produced by the highcurrent, highly-compressed electron beam.

\section{lonization time}

The ionization time for stripping singly charged radioactive ions to high charge states is important for two reasons: (1) The ion beam intensity will be reduced for isotopes with lifetimes less than the ionization time, and (2) the ionization rate sets an upper limit on the highlycharged-ion production rate. In an EBIT, ionization to high charge states proceeds primarily by sequential single ionization. The characteristic tine for each ionization step is $\tau_{i}=1 / \sigma_{i} \varphi_{e}$, where $\sigma_{i}$ is the ionization cross section and $\varphi_{e}$ is the electron flux. The total time to remove $N$ electrons is then $t_{N}=\sum_{i=1}^{N} \tau_{i}$. Wc used Lotz-formula cross sections ( 7 ) to calculate the ionization times for tin ( $Z$ $=50$ ) ions at $30-\mathrm{keV}$ electron energy. The value of the electron flux used was $\varphi_{\mathrm{e}}=3.4 \times 10^{23} \mathrm{~cm}^{-2} \mathrm{~s}^{-1}$. This corresponds to the average electron current density for electron and ion radial distributions that have a Gaussian profile with the same characteristic radius, and with highintensity-EBIT parameters as given in Table $I$.

The total time required to reach the different charge states of tin is plotted in Fig. 3. The time required to ionize tin isotopes from $q=1+$ to $q=40+$ (Ne-like) and $q=$ $48+$ (He-like) is $3.0 \mathrm{~ms}$ and $34 \mathrm{~ms}$, respectively. These closed shell configurations are favorable because the jump in ionization potential between shells results in a narrower charge state distribution. In fact, $30 \mathrm{keV}$ is below the Kshell ionization potential for tin; hence the ionization stops at $q=48+$, and that charge state will have a high abundance after $34 \mathrm{~ms}$. This effect could be used to improve the purity of a ${ }^{100} \mathrm{Sn}$ beam by removing contaminating ${ }^{100} \mathrm{In}(Z=49)$ ions, for which $q=47+$ is the highest possible charge state in a $30-\mathrm{keV}$ electron beam.

The ionization time can be used to calculate an upper limit for the highly-charged-ion output of the high intensity EBIT used as a charge state booster. If every highly charged ion could be removed as soon at it was produced, the ion production rate would be $N_{i} / t_{q}$, where $N_{i}$ is the ion capacity of the trap and $l_{q}$ is the ionization time to reach charge state $q$. Assuming $q=40+$ and $50 \%$ neutralization of the electron space charge by ions, $N_{i}=1.0 \times 10^{9}$ ions. The upper limit for the production rate of $\mathrm{Sn}^{40+}$ ions is then
$3.3 \times 10^{11}$ ions/s. The actual production rate will be lower and is difficult to calculate, but it is probably between $10^{10}$ and $10^{11}$ ions/s.

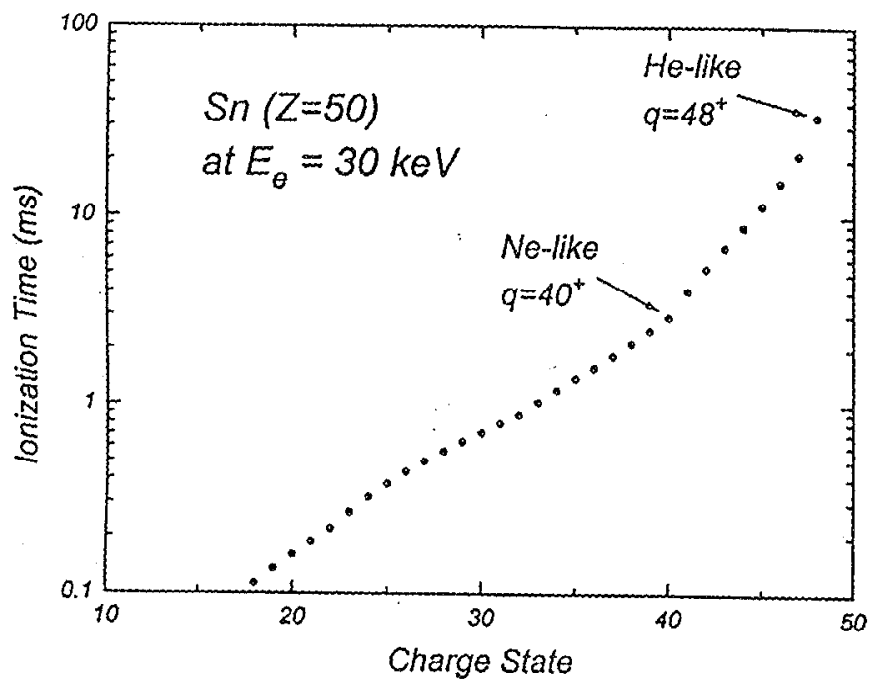

FIGURE 3. Calculated total ionization time to reach the different charge states of tin in a high intensity EBIT.

\section{Charge state distribution}

The charge state distribution of the highly charged radioactive ions extracted from an EBIT charge state booster is important because, as with foil stripping, only one charge state is likely to be accelerated and the others will be lost. The charge state distribution in an EBIT is determined by a combination of ionization, recombination, and loss processes. Measurements from existing EBIT and EBIS devices provide a good indication of what can be expected. An abundance of $90 \%$ for the $q=46+$ (Ne-like) charge state of barium has been observed in an EBIT (1). Away from closed shells, an abundance of roughly $25 \%$ was observed for the most abundant $(q=77+)$ charge state in a beam of thorium ions extracted from an EBIT (8). EBIS measurements, available for lighter elements such as argon, show similar distributions (9). Thus, experience with existing EBIT and EBIS devices suggests that an ionization balance with $25 \%$ or more of the ions in the most abundant charge state can be expected for RIB applications.

\section{Emittance}

The stripping of accelerated ions to high charge states with foils or gas can only increase the emittance of the beam. In marked contrast, stripping of ions in an EBIT reduces the beam emittance. Rough measurements of the emittance of beams from existing EBITs and computer modeling suggest that an absolute emittance of roughly $1 \pi$ 
mm mrad at $30 \mathrm{kV}$ can be expected for the LLNL high intensity EBIT $(10,11)$. One contribution to the enittance reduction can be seen from the explicit $q$ dependence of Eq. (1). However, this is offset by the Coulomb energy gained by ions as they move toward the beam axis and by electron beam heating of very-highly-charged ions held for long times in the trap. Evaporative ion-ion cooling, in which light (stable) ions are introduced into the trap as a coolant, is used to control the temperature and emittance of highly charged ions in EBIT devices (2).

The decrease in ion emittance in the EBIT has a favorable impact on a RIB post-accelerator. For example, beam losses and radioactive contamination would be reduced, the resolving power of a mass separator would be improved, and RIBs could be focused to micron-size spots.

\section{Duty cycle}

Ions can be injected into an EBIT either continuously, at an energy just above the axial trap barrier, or in bunches by lowering the trap barrier during the arrival of the ion bunch. With continuous injection, all injected ions not ionized to higher charge in one transit of the trap will escape the way they entered. Returning to the example of tin, the ionization time for $\mathrm{Sn}^{1+}$ ions in the high intensity EBIT is $0.16 \mu \mathrm{s}$, a factor of 100 less than the $16 \mu \mathrm{s}$ bounce time of $500-\mathrm{eV}$ tin ions in a $25-\mathrm{cm}$ trap. Hence, $100 \%$ ionization and capture can be achieved with either injection mode. Note that this is not true for EBIS devices that use low electron beam current densities of order $200 \mathrm{~A} / \mathrm{cnu}^{2}$.

Charge-boosted ions can be extracted either continuously by allowing them to leak out over the axial trap barrier, or in batches $(12,13)$. Using batch mode, the EBIT can function as an ion accumulator so that a pulsed RFQ and LINAC can be used as a post-accelerator. Note that the $3.0-\mathrm{ms}$ ionization time to produce $\mathrm{Sn}^{40+}$ inns is compatible with a $120 \mathrm{~Hz}$ repetition rate.

\section{IMPACT ON RIB FACILITY}

The use of an EBIT for charge state boosting will have a large impact on the post-accelerator that follows it. For example, an estimate of the post-acceleration required to accelerate radioactive ${ }^{132} \mathrm{Sn}$ ions to an energy of $7 \mathrm{MeV} / \mathrm{u}$ with two stages of conventional stripping indicates that a total accelerating potential of $77 \mathrm{MV}$ is required, and that the expected stripping efficiency is $8 \%$ (14). If the ions were stripped to $q=40+$ in an EBIT before acceleration, then only $24 \mathrm{MV}$ of acceleration potential would be required.

The REX-ISOLDE project at CERN is using a large Penning trap with buffer gas cooling as an accumulator and emittance reducer for singly charged ions. The ions will be transferred to an EBIS in batch mode for stripping to higher charge states. The capacity of the Penning trap is expected to limit the ion throughput to $\sim 10^{7}$ ions/s (15). The estimates presented here indicate that a high intensity EBIT does not require a Penning trap accumulator.

\section{CONCLUSIONS AND FUTURE PLANS}

The estimates presented here indicate that a high intensity EBIT under development at LLNL is an attractive option for charge state boosting of radioactive ion beams in an ISOL-type RIB facility. Other advantages of the EBIT are a significant reduction in the ion beam emittance and the separation of interfering isobars in some cases. These features will facilitate cleaner beams and reduce losses.

An experimental verification of the performance characteristics presented here is crucial for an evaluation of options for a future RIB facility. We propose to use ISOLtype sources for injecting ions into a high intensity EBIT at LLNL in order to obtain measurements of the performance characteristics presented here.

\section{ACKNOWLEDGMENT}

This work was performed under the auspices of the $U$. S. Department of Energy by Lawrence Livermore National Laboratory under Contract No. W-7405-Eng-48.

\section{REFERENCES}

1. R. E. Marrs, M. A. Levine, D. A. Knapp, and J. R. Henderson, Phys. Rev. Lett. 60, 1715 (1988).

2. M. A. Levine, R. E. Marrs, J. R. Henderson, D. A. Knapp, and M. B. Schneider, Phys. Scr. T22, 157 (1988).

3. E. D. Donets in The Physics and Technology of Ion Sources, edited by I. G. Brown (John Wiley, New York, 1989), p. 245.

4. D. A. Knapp, R. E. Marrs, S. R. Elliott, E. W. Magee, and R. Zasadzinski, Nucl. Instrum. Meth. A 334, 305 (1993).

5. R. Keller in The Physics and Technology of Ion Sonrces, edited by I. G. Brown (John Wiley, New York, 1989), p. 23.

6. U. Köster, O. Kester, and D. Habs, Rev. Sci. Instrum. 69, $1316(1998)$.

7. W. Lotz, Z. Phys. 216, 241 (1968).

8. D. Schneider, M. W. Clark, B. M. Penetrante, J. McDonald, D. DeWitt, and J. N. Bardsley, Phys. Rev. A 44, 3119 (1991).

9. B. Visentin, P. Van Duppen, P. A. Leroy, F. Hanaul, and R. Gobin, Nucl. Instrum. Meth. B 101, 275 (1995).

10. R. E. Marrs, Nucl. Instrum. Meth. $B$ (in press).

11. R. E. Marrs, D. H. Schneider, and J. W. McDonald, Rev. Sci. Instrum. 69, 204 (1998).

12. M. P. Stockli, Rev. Sci. Instrum. 67, 892 (1996).

13. L. P. Ratliff, E. W. Bell, D. C. Parks, A. I. Pikin, and J. D. Gillaspy, Rev. Sci. Instrum. 68, 1998 (1997).

14. J. A. Nolen, Rev. Sci. Instrum. 67, 935 (1996); Concept for an Advanced Exotic Beam Facility Based on $A T L A S$, edited by E. Rehm (Physics Division, Argonne National Laboratory, 1995).

15. D. Habs et al., Nucl. Phys. A616, 29c (1997). 